\title{
Regular coffee intake improves liver enzyme levels and liver histology in patients with chronic alcohol consumption, non-alcoholic fatty liver and non-alcoholic steatohepatitis: Report of 259 cases
}

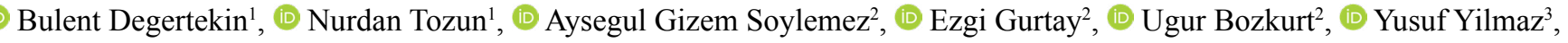 \\ (1) Suna Yapali ${ }^{1}$, (1) Eser Vardareli ${ }^{1}$, (1) Hakan Umit Unal ${ }^{1}$, (1) Berkan Colakoglu², (1) Can Berk Alpaydin² \\ ${ }^{1}$ Department of Gastroenterology and Hepatology, Acibadem Mehmet Ali Aydinlar University School of Medicine, Istanbul, Turkey; ${ }^{2}$ Acibadem Mehmet Ali Aydinlar \\ University School of Medicine, Istanbul, Turkey; ${ }^{3}$ Department of Gastroenterology and Hepatology, Marmara University, School of Medicine, Istanbul, Turkey
}

\begin{abstract}
Background and Aim: This study aims to investigate the effects of chronic coffee consumption ( $>5$ years) and type of coffee in non-alcoholic steatohepatitis (NASH), non-alcoholic fatty liver (NAFLD) and patients who have regular alcohol consumption.

Materials and Methods: In this study, 158 healthy individuals and 101 patients with histologically proven NASH were enrolled. The daily amount of coffee intake, amount of alcohol use and type of coffee were calculated for all patients. The degree of steatosis and fibrosis was analyzed by transient elastography and liver ultrasound in non-NASH and by liver biopsy in NASH patients.

Results: Patients with a history of coffee consumption $(n=132)$ had lower liver enzyme levels compared to the non-coffee group $(n=127)(p=0.001)$. Serum ALT level was significantly lower [ALT: $21.2 \pm 11.7$ U/L vs. $56.4 \pm 15.6$ $\mathrm{U} / \mathrm{L}(\mathrm{p}=0.004)]$, and the liver histopathology was significantly better for patients with a coffee consumption of daily for $>5$ years $(\mathrm{p}=0.045$ for fibrosis score for $\mathrm{NASH}, \mathrm{p}=0.036$ for $\mathrm{LSM}$ and $\mathrm{p}=0.015$ for CAP measurements for the non-NASH patient).

Conclusion: Coffee seems to have a positive protective effect on liver histology and liver enzyme levels in healthy individuals, in patients with chronic alcohol consumption, NAFLD and NASH. These results are more prominent in patients who drink coffee on a regular daily base for more than five years.
\end{abstract}

Keywords: Alcohol; coffee; fatty liver.

How to cite this article: Degertekin B, Tozun N, Soylemez AG, Gurtay E, Bozkurt U, Yilmaz Y, et al. Regular Coffee intake improves liver enzyme levels and liver histology in patients with chronic alcohol consumption, non-alcoholic fatty liver and non-alcoholic steatohepatitis: Report of 259 cases. Hepatology Forum 2020; $1(3): 88-96$.

Received: September 14, 2020; Accepted: September 18, 2020; Available online: September 21, 2020

Corresponding author: Bulent Degertekin; Department of Gastroenterology, Acibadem Ankara Hospital, Ankara, Turkey

Phone: +90 31259343 96; e-mail: bulent.degertekin@acibadem.com.tr

(c) (\$) OPEN ACCES

OPEN ACCESS

(C) Copyright 2020 by Hepatology Forum - Available online at www.hepatologyforum.org

\section{Introduction}

Coffee is a widely-consumed beverage, which is mostly preferred for its stimulatory effects and taste. Recent data have shown that it has beneficial effects on general health and affects the course and the outcome of liver diseases. In a recent meta-analysis published by Freedman et al., ${ }^{[1]}$ a dose-dependent inverse association between coffee consumption and total mortality was described, where men and women who drank six or more cups daily had an $10 \%$ and $15 \%$ decreased risk of death. Although a few studies which were published earlier did not find any significant effect of coffee on liver steatosis and non-alcoholic steatohepatitis (NASH),${ }^{[2-4]}$ most of the studies published in the past decade suggest that coffee has a beneficial effect on the course and the outcomes of liver diseases. Case-control studies from Japan and Italy and prospectively designed studies from Japan and Finland reported a dose-dependent inverse association between coffee and hepatocellular cancer incidence. ${ }^{[5-8]}$ Studies designed from China, Japan and the USA also reported that chronic HBV and HCV cases that drank coffee had decreased fibrosis stage and increased virological response to interferon treatment. ${ }^{[2,9]}$ Recently, published studies have also shown that coffee reduced the risk of steatosis and had a significant impact on the amount of fibrosis and steatosis in patients with NASH. ${ }^{[10-13]}$

This hepatoprotective effect of coffee in NASH and non-alcoholic fatty liver (NAFLD) patients is mostly mediated by its antioxidant (reduces reactive oxygen species and oxidized glutathione level), metabolic (increase in adiponectin, decrease in serum leptin, and saturated fatty acid level), anti-inflammatory (decrease of TNF-alpha, IFN-gamma, PPAR-alpha, IL-4 and IL-10) and anti-fibrotic (decrease of TGF-beta, connective tissue growth factor) effects. Cancer preventive effect has been linked to its ingredients, such as cafestol and kahweol and mediated by stimulation of ARE signaling, inhibition of phase I activating enzyme activation and induction of phase II detoxifying enzymes, such as glutathione S-transferase. ${ }^{[14-17]}$

The effects of coffee on alcoholic liver disease have not yet been investigated thoroughly. The presentation of alcoholic liver disease may vary from simple steatosis to inflammation with hepatocellular necrosis, liver fibrosis and cirrhosis. The aforementioned protective effects of coffee can also be applied to alcoholic liver diseases. Hu et al. ${ }^{[7]}$ 
recently published a study where they collected the data of 60,323 individuals and analyzed the effects of coffee consumption on GGT levels in patients who had alcohol consumption. The data clearly showed that especially in men, the elevation of GGT induced by heavy drinking ( $>280 \mathrm{~g} /$ week) was significantly reduced by coffee consumption exceeding four cups per day.

Coffee can be drip-brewed, filtered, French pressed, percolated or boiled as in Turkish coffee. Studies have shown that caffeine levels in a cup of coffee vary considerably due to variations in coffee blend, exact amount of ground coffee used, and the brewing technique. The amount of caffeine may vary from 107 to $200 \mathrm{mg}$ per $8 \mathrm{fl}$. oz. A cross-sectional study from France, which included 195 patients, reported that regular coffee but not espresso was protective against liver fibrosis in patients with NAFLD. Studies investigating the effects of different types of coffee on different types of liver diseases are lacking. ${ }^{[3]}$

In this study, we aimed to investigate whether chronic coffee consumption ( $>5$ years) and type of coffee had a beneficial effect on liver histology and liver enzyme levels in different groups, such as healthy individuals with normal liver findings, patients with NASH or NAFLD and patients who had regular alcohol consumption.

\section{Materials and Methods}

\section{Patient Population}

One hundred and fifty-eight healthy individuals from the check-up unit and 101 patients with histologically proven NASH were enrolled in this study. The healthy individuals were enrolled at the Acrbadem Health Group Hospitals (7 hospitals in Istanbul) and patients with NASH were enrolled at the Gastroenterology and Hepatology Unit of Marmara University School of Medicine in turkey. The data of the assumed healthy individuals were collected prospectively between 2012 and 2018. The data of the patients with NASH were collected at the outpatient clinic at the time when they were diagnosed with NASH via liver biopsy or when they visited the University of Marmara Division of Gastroenterology for routine follow-up. Healthy individuals were divided into two major groups based on the presence of liver steatosis on ultrasound ( $\mathrm{n}=85$ normal liver ultrasound findings, $\mathrm{n}=73$ steatosis of the liver); patients with a normal ultrasound finding was defined as a healthy individual with normal liver findings meanwhile patients with steatosis on liver ultrasound were defined as a healthy individual with liver steatosis.

Demographics, clinical, laboratory (blood counts, creatinine, liver panel, hepatitis B and C serology), and radiologic (liver ultrasound) data, as well as the amount of daily coffee intake and the type of coffee, and the amount of alcohol use, were noted and body mass index (BMI) was calculated for all patients. The degree of steatosis and fibrosis was analyzed using both liver ultrasound transient elastography (TE) in all patients and by liver biopsy in patients with NASH. NAFLD was defined as a demonstration of hepatic steatosis in the liver by ultrasound in the absence of significant alcohol use ( $>140 \mathrm{gr} /$ week for women and $>210 \mathrm{gr}$ /week for men) and exclusion of chronic liver disease. NASH was defined by liver biopsy findings, where the specimens were scored according to steatosis, activity, and fibrosis by a pathologist expertized in the liver. ${ }^{[18]}$

Patients with a history of potentially hepatotoxic drug usage, obesity surgery, viral hepatitis (B, C and D), cholestatic liver disease, hemochromatosis, drug-induced liver disease and Wilson's disease were excluded from this study.

\section{Collection of Data and Definitions}

Coffee intake and other dietary information were obtained using a Quantitative Food Frequency Questionnaire. The question about coffee read, "How many cups of coffee do you usually drink per day?" with preset categories: "Do not drink coffee", "1-2 cups", "3-4 cups", and "5 or more cups". The type of coffee was also questioned as Turkish, granulated, filtered and combination of these three options. Alcohol consumption was recorded using two questions: "Do you regularly drink alcohol?" Drinkers of alcohol were defined as people who answered yes or no. The second question was, "How much alcohol do you drink per week?" This was an open-ended question and participants specified the type and amount of alcohol they take per week. The amount of alcohol was later calculated as grams/per week for each participant. Regular alcohol consumption was defined as taking $>210 \mathrm{gr} /$ week for men and >140 gr/week for women. Patients were classified into subgroups as follows: people who reported no alcohol consumption were referred to as non-drinkers, patients who had alcohol intake of $<210 \mathrm{gr} /$ week for men and $<140 \mathrm{gr} /$ week for women were referred to as mild drinkers, 210-420 gr/week for men and 140-280 gr/week for women as moderate drinkers and $>420 \mathrm{gr} /$ week for men and $>280 \mathrm{gr} /$ week for women as heavy drinkers. Although the upper limit of normal for serum ALT has been a topic of debate in recent years and according to a very recent study, the upper limit of normal for serum ALT has been determined as $32 \mathrm{U} / \mathrm{L}$ for men, $23 \mathrm{U} / \mathrm{L}$ for women in healthy Turkish population. ${ }^{[19]}$ Normal serum ALT levels in this study were picked as 40 $\mathrm{U} / \mathrm{L}$ for both genders.

To understand the impacts of duration of coffee consumption, patients were also divided into four groups based on the amount and duration of the coffee they drank. Patients who did not drink any coffee were defined as non-coffee drinkers; patients who drank coffee occasionally (not daily) were defined as occasional coffee drinkers; patients who drank coffee on a regular basis -daily for less than five years were defined as regular coffee drinkers ( $<5$ years), meanwhile patients who had a history of daily coffee consumption for more than five years were defined as regular coffee drinkers ( $>5$ years).

The effects of chronic coffee consumption ( $>5$ years) and type of coffee (granulated, decaffeinated and Turkish coffee) on liver histology and liver function tests were analyzed in patients with NASH, fatty liver, and patients who had regular alcohol intake.

The Institutional Review Board at the participating center approved this study and written informed consent was obtained from all patients before study entry.

\section{Transient Elastography}

A single operator performed all TE examinations according to the manufacturer's instruction. The examination was performed over the right lateral intercostal spaces with the patient lying in the dorsal decubitus position and the right arm being in maximal abduction. The tip of the transducer probe was placed on the skin between the ribs at the $7-8^{\text {th }}$ intercostal space in the mid or anterior axillary line over the right lobe of the liver. LSM (liver stiffness measure) assessment and CAP (controlled attenuation parameter) was performed on a FibroScan 502 touch (Echosens SA, Paris, France) using the M probe as described previously. ${ }^{[20]}$ The XL probe was used for obese patients. Patients with LSM failures or unreliable examinations were excluded from this study. LSM failure was defined as zero valid shots, and unreliable examinations were defined as fewer than 10 valid shots, an interquartile range/LSM 
Table 1. Characteristics of the study population

\begin{tabular}{|c|c|c|c|c|c|}
\hline & $\begin{array}{l}\text { Healthy individuals } \\
\text { (no steatosis) } \\
(n=85)\end{array}$ & $\begin{array}{l}\text { Healthy individuals } \\
\text { (with steatosis) } \\
\text { ( } n=73)\end{array}$ & $\begin{array}{l}\text { NASH } \\
(n=101)\end{array}$ & $\begin{array}{l}\text { Total } \\
(n=259)\end{array}$ & $\mathbf{p}$ \\
\hline Age $(Y)$ & $42.4 \pm 10.3$ & $43.5 \pm 9.1$ & $45.0 \pm 11.6$ & $44.1 \pm 11.3$ & 0.102 \\
\hline $\operatorname{Sex}[M(\%)]$ & $39(45.8)$ & $36(49.3)$ & $50(49.5)$ & $125(48.2)$ & 0.255 \\
\hline $\mathrm{BMI}\left(\mathrm{kg} / \mathrm{m}^{2}\right)$ & $24.4 \pm 4.3$ & $26.3 \pm 6.7$ & $27.1 \pm 5.3$ & $26.4 \pm 5.9$ & 0.091 \\
\hline \multicolumn{6}{|l|}{ Laboratory } \\
\hline ALT (U/L) & $26.3 \pm 11.2$ & $45.4 \pm 13.3$ & $55.7 \pm 10.0$ & $46.4 \pm 12.6$ & 0.022 \\
\hline AST (U/L) & $22.0 \pm 12.1$ & $37.1 \pm 15.7$ & $40.6 \pm 13.1$ & $38.4 \pm 14.5$ & 0.059 \\
\hline GGT (U/L) & $55.0 \pm 12.4$ & $78.1 \pm 17.1$ & $91.6 \pm 16.4$ & $81.8 \pm 15.6$ & 0.034 \\
\hline HOMA-IR & $1.9 \pm 0.3$ & $2.2 \pm 0.9$ & $3.9 \pm 1.1$ & $2.8 \pm 1.1$ & 0.012 \\
\hline Daily amount of coffee consumption & & & & & 0.015 \\
\hline None & $10(11.7)$ & $41(56.2)$ & $76(75.4)$ & $127(49.0)$ & \\
\hline$<1$ cup & $12(14.2)$ & $6(8.2)$ & $10(9.9)$ & $28(10.9)$ & \\
\hline $1-2$ cup & $19(22.4)$ & $13(17.8)$ & $9(8.9)$ & $41(15.9)$ & \\
\hline $3-4$ cup & $21(24.7)$ & $9(12.4)$ & $3(2.9)$ & $33(12.6)$ & \\
\hline$>4$ cup & $23(27.0)$ & $4(5.4)$ & $3(2.9)$ & $30(11.6)$ & \\
\hline Duration and amount of coffee consumption & & & & & 0.028 \\
\hline Occasional coffee drinker & $19(22.4)$ & $13(17.8)$ & $14(13.8)$ & $46(17.8)$ & \\
\hline Regular coffee drinker (<5 yr) & $23(27.1)$ & $4(5.4)$ & $6(5.9)$ & $33(12.8)$ & \\
\hline Regular coffee drinker ( $>5 \mathrm{yr}$ ) & $33(38.8)$ & $15(20.6)$ & $5(4.9)$ & $53(20.4)$ & \\
\hline Alcohol consumption & & & & & 0.178 \\
\hline None & $51(60.0)$ & $52(71.2)$ & $101(100)$ & $204(78.7)$ & \\
\hline Mild & $29(34.2)$ & $6(8.4)$ & 0 & $35(13.5)$ & \\
\hline Moderate & $3(3.5)$ & $8(10.9)$ & 0 & $11(4.4)$ & \\
\hline Heavy & $2(2.3)$ & $7(9.5)$ & 0 & $9(3.42)$ & \\
\hline
\end{tabular}

NASH: Non-alcoholic steatohepatitis; Y: Year; M: Male; BMI: Body mass index. Results expressed as number (\%) or median (range) unless specified otherwise. P-value for comparison of all groups.

more than $30 \%$, or a success rate less than $60 \%$. A reliable LSM result was defined as at least 10 valid shots, a success rate of at least $60 \%$, and an interquartile range of less than $30 \%$ of the median LSM value. Results were considered unreliable if these criteria were not fulfilled. ${ }^{[20,21]}$ Metavir fibrosis score of F0-F1, F2, F3 and F4 were defined as LSM of $\leq 7 \mathrm{kPa}, 7.1-9.9 \mathrm{kPa}, 10-12.9 \mathrm{kPa}$ and $\geq 13 \mathrm{kPa}$, respectively. Steatosis was graded as the percentage of hepatocytes with fat: S0, $<5 \%$; S1, $5-33 \%$; S2, 34-66\%; S3, $>66 \%$. Cut-offs of CAP were defined as 215 $\mathrm{dBm}$ for $\geq \mathrm{S} 1,252 \mathrm{dBm}$ for $\geq \mathrm{S} 2$ and $296 \mathrm{dBm}$ for $\mathrm{S} 3 .{ }^{[22,23]}$

\section{Statistical Analysis}

Categorical data were presented as number and percent and were compared using the chi-square test or Fisher's exact test as appropriate. Continuous variables were expressed as mean and standard deviation (SD) unless specified otherwise, and compared using t-test or Mann-Whitney U-test. To analyze the effects of coffee consumption on liver function tests in patients who had regular alcohol consumption, variables with a p-value of $<0.1$ on univariate analysis were entered into a Cox regression hazards model by forward logistic regression. By this way, independent predictors of elevated liver enzymes in patients who had regular alcohol consumption were analyzed. All statistical analyses were performed using SPSS v. 14.0.8 statistical software (SPSS Inc. Chicago, IL).

\section{Results}

\section{Characteristics of the Patients}

In this study, 259 patients, including $101 \mathrm{NASH}, 73$ healthy individuals with steatosis on liver ultrasound and 85 healthy individuals with normal liver on ultrasound, were included. Characteristics of the study population are summarized in Table 1.

Healthy individuals with normal liver findings were younger, but there was no difference in age between the groups, with mean ages in the three groups being $42.4 \pm 10.3,43.5 \pm 9.1$ and $45.0 \pm 11.6$ years, respectively. This group of patients also had lower BMI and serum ALT levels compared to individuals with liver steatosis and NASH: $24.4 \pm 4.3$ vs. $26.3 \pm 6.7$ and $27.1 \pm 5.3$ respectively for BMI $(\mathrm{p}>0.05)$ and $26.3 \pm 11.2 \mathrm{U} / \mathrm{L}$ vs. $45.4 \pm 13.3 \mathrm{U} / \mathrm{L}$ and $55.7 \pm 10.0 \mathrm{U} / \mathrm{L}$ for serum ALT level $(\mathrm{p}=0.022)$. The amount of coffee consumption was significantly higher in healthy individuals with normal liver findings than individuals with liver steatosis and patients with NASH $88.3 \%$ vs. $43.8 \%$ vs. $24.6 \%$, respectively $(\mathrm{p}=0.015)$. Meanwhile, there were more patients with a history of moderate to heavy alcohol consumption in healthy individuals with liver steatosis, $20.4 \%$ vs. $5.8 \%$, but this difference was not significant $(\mathrm{p}=0.178)$ (Table 1$)$.

\section{Effects of Coffee on Liver Enzymes and Liver Histology}

Patients who had a coffee drinking history $(n=132)$ had lower liver en- 
Table 2. The effects of coffee consumption on liver enzyme levels and liver histology

\begin{tabular}{|c|c|c|c|c|c|c|}
\hline & $\begin{array}{l}\text { Non-coffee } \\
\text { drinker } \\
(n=127)\end{array}$ & $\begin{array}{l}\text { Occasional } \\
\text { drinker } \\
(n=46)\end{array}$ & $\begin{array}{l}\text { Regular drinker } \\
(<5 \text { yr }) \\
(n=33)\end{array}$ & $\begin{array}{l}\text { Regular drinker } \\
(>5 \text { yr) } \\
(n=53)\end{array}$ & $\begin{array}{l}\text { Total } \\
(n=259)\end{array}$ & $\mathbf{p}$ \\
\hline Sex $[M(\%)]$ & $64(50.3)$ & $24(52.1)$ & $15(45.4)$ & $55(43.3)$ & $125(48.2)$ & 0.518 \\
\hline $\mathrm{BMI}\left(\mathrm{kg} / \mathrm{m}^{2}\right)$ & $26.9 \pm 6.9$ & $26.5 \pm 6.6$ & $25.1 \pm 5.8$ & $25.9 \pm 5.4$ & $26.4 \pm 5.9$ & 0.255 \\
\hline \multicolumn{7}{|l|}{ Liver enzymes } \\
\hline AST (U/L) & $42.6 \pm 15.2$ & $32.9 \pm 14.9$ & $25.2 \pm 11.6$ & $19.5 \pm 10.4$ & $38.4 \pm 14.5$ & 0.030 \\
\hline GGT (U/L) & $94.2 \pm 16.7$ & $79 \pm 13.6$ & $63.1 \pm 15.9$ & $51.1 \pm 11.6$ & $81.8 \pm 15.6$ & 0.050 \\
\hline Steatosis on liver USG & $101 / 127(79.5)$ & $30 / 46(65.2)$ & $16 / 33(54.5)$ & $23 / 53(43.3)$ & $170 / 259(65.6)$ & 0.042 \\
\hline \multicolumn{7}{|c|}{ TE findings for Non-NASH } \\
\hline LSM (kPa) & $6.05 \pm 3.86$ & $5.97 \pm 2.93$ & $5.80 \pm 2.98$ & $4.98 \pm 2.06$ & $5.92 \pm 3.09$ & 0.036 \\
\hline
\end{tabular}

Y: Year; M: Male; BMI: Body mass index; USG: Ultrasonography; TE: Transient elastography; NASH: Non-alcoholic steatohepatitis; LSM: Liver stiffness measure; CAP: Controlled attenuation parameter; NAS: NAFLD activity score. Results expressed as number (\%) or median (range) unless specified otherwise. P-value for comparison of all groups.

Table 3. The effects of coffee consumption on liver histology in NASH patients

\begin{tabular}{|c|c|c|c|c|c|c|}
\hline & $\begin{array}{l}\text { Non-coffee } \\
\text { drinker } \\
(n=76)\end{array}$ & $\begin{array}{l}\text { Occasional } \\
\text { drinker } \\
(n=14)\end{array}$ & $\begin{array}{l}\text { Regular drinker } \\
(<5 \mathrm{yr}) \\
(\mathrm{n}=6)\end{array}$ & $\begin{array}{l}\text { Regular drinker } \\
(>5 \mathrm{yr}) \\
(\mathrm{n}=5)\end{array}$ & $\begin{array}{l}\text { Total } \\
(n=101)\end{array}$ & p \\
\hline Age $(Y)$ & $47.6 \pm 10.2$ & $44.0 \pm 8.4$ & $44.3 \pm 10.1$ & $43.9 \pm 8.2$ & $45.0 \pm 11.6$ & 0.304 \\
\hline $\operatorname{Sex}[M(\%)]$ & $28(52.8)$ & $6(42.8)$ & $3(50.0)$ & $3(60.0)$ & $50(49.5)$ & 0.276 \\
\hline $\mathrm{BMI}\left(\mathrm{kg} / \mathrm{m}^{2}\right)$ & $26.9 \pm 7.6$ & $26.0 \pm 6.3$ & $26.3 \pm 6.1$ & $25.9 \pm 5.6$ & $27.1 \pm 6.9$ & 0.166 \\
\hline \multicolumn{7}{|l|}{ Liver enzymes } \\
\hline ALT (U/L) & $66.1 \pm 14.6$ & $48.2 \pm 11.5$ & $39.3 \pm 12.1$ & $30.4 \pm 9.2$ & $55.7 \pm 13.8$ & 0.028 \\
\hline AST (U/L) & $55.1 \pm 13.9$ & $43.0 \pm 9.9$ & $38.7 \pm 13.1$ & $18.8 \pm 12.6$ & $42.6 \pm 12.7$ & 0.042 \\
\hline GGT (U/L) & $95.4 \pm 15.6$ & $88 \pm 12.1$ & $88 \pm 11.8$ & $83.8 \pm 9.8$ & $91.6 \pm 13.9$ & 0.342 \\
\hline \multicolumn{7}{|l|}{ Liver histology } \\
\hline NAS score & $5.91 \pm 0.86$ & $5.24 \pm 0.54$ & $5.32 \pm 1.01$ & $5.01 \pm 0.41$ & $5.78 \pm 0.95$ & 0.098 \\
\hline Fibrosis score & $2.03 \pm 0.42$ & $1.92 \pm 0.19$ & $1.04 \pm 0.36$ & $0.80 \pm 0.12$ & $1.95 \pm 0.41$ & 0.045 \\
\hline
\end{tabular}

NASH: Non-alcoholic steatohepatitis; Y: Year; M: Male; BMI: Body mass index; NAS: NAFLD activity score. Results expressed as number (\%) or median (range) unless specified otherwise. P-value for comparison of all groups.

zyme levels compared to those who did not drink any coffee $(\mathrm{n}=127)$ [ALT: $29.8 \pm 10.9 \mathrm{U} / \mathrm{L}$ vs. $56.4 \pm 15.6 \mathrm{U} / \mathrm{L}(\mathrm{p}=0,001)$, AST: $24.9 \pm 10.8$ $\mathrm{U} / \mathrm{L}$ vs.42.6. $\pm 15.2 \mathrm{U} / \mathrm{L}(\mathrm{p}=0.032)]$. The study group was divided into four subgroups based on the amount and duration of coffee consumption as non-coffee drinkers $(n=127)$, occasional coffee drinkers $(n=46)$, regular coffee drinkers for less than five years $(n=33)$, and regular coffee drinkers for more than five years $(n=53)$. Age, gender, and BMI were comparable among the four groups $(\mathrm{p}>0.005)$ (Table 2$)$.

Mean serum ALT and AST levels were $21.2 \pm 11.7 \mathrm{U} / \mathrm{L}$ and $19.5 \pm 10.4$ $\mathrm{U} / \mathrm{L}$ for the regular coffee drinkers ( $>5$ years) group, $56.4 \pm 15.6 \mathrm{U} / \mathrm{L}$ and $42.6 \pm 15.2 \mathrm{U} / \mathrm{L}$ for non-coffee drinkers, $38.7 \pm 11.1 \mathrm{U} / \mathrm{L}$ and $32.9 \pm 14.9$ $\mathrm{U} / \mathrm{L}$ for occasional coffee drinkers, and $30.3 \pm 9.2 \mathrm{U} / \mathrm{L}$ and $25.2 \pm 11.6$ $\mathrm{U} / \mathrm{L}$ for regular coffee drinkers $(<5$ years) group. Mean serum ALT, AST and GGT levels were significantly lower in regular coffee drinkers for more than five years' group compared to non-coffee drinkers group $(\mathrm{p}=0.004$ for ALT, $\mathrm{p}=0.030$ for AST and $\mathrm{p}=0.050$ for GGT) (Table 2).
TE findings showed that mean LSM and CAP score was significantly lower in the regular coffee drinkers ( $>5$ years) group compared to other groups ( $\mathrm{p}=0.036$ for LSM score and $\mathrm{p}=0.015$ for CAP score). LSM scores for non-coffee drinkers, occasional drinkers' regular drinkers $<5$ years and regular drinkers $>5$ years were; $6.05 \pm 3.86$ (5.2-14.8), 5.97 \pm 2.93 (4.0-11.3), 5.80 \pm 2.98 (4.6-12.1) and 4.98 \pm 2.06 (3.1-7.0), respectively. Meanwhile the CAP scores were $250.52 \pm 86.2$, $232.96 \pm 79.36,201.79 \pm 64.11179 .02 \pm 66.03$ respectively (Table 2 ).

\section{Effect of Coffee on Liver Enzymes and Liver Histology in Patients with NASH}

The effects of coffee consumption on liver histology were analyzed in 101 patients with NASH. Among these patients, 76 were non-coffee drinkers, 14 were occasional coffee drinkers, six were regular coffee drinkers for less than five years and five were regular coffee drinkers for more than five years. Patients with NASH who drank 
Table 4. The effects of coffee type on liver enzymes and liver histology

\begin{tabular}{|c|c|c|c|c|c|c|}
\hline & $\begin{array}{l}\text { Turkish } \\
(n=31)\end{array}$ & $\begin{array}{l}\text { Granulated } \\
(n=6)\end{array}$ & $\begin{array}{l}\text { Filtered } \\
(n=2)\end{array}$ & $\begin{array}{l}\text { Combination } \\
(n=93)\end{array}$ & $\begin{array}{l}\text { Total } \\
(n=132)\end{array}$ & $\mathbf{p}$ \\
\hline Age $(Y)$ & $48.0 \pm 10.6$ & $42.0 \pm 9.4$ & $40.2 \pm 12.1$ & $42.4 \pm 9.9$ & $43.8 \pm 11.1$ & 0.409 \\
\hline $\mathrm{BMI}\left(\mathrm{kg} / \mathrm{m}^{2}\right)$ & $25.2 \pm 4.1$ & $25 \pm 4.6$ & $27.1 \pm 5.0$ & $26.8 \pm 6.4$ & $26.1 \pm 5.8$ & 0.206 \\
\hline \multicolumn{7}{|l|}{ Liver enzymes } \\
\hline Steatosis on liver USG & $15(48.3)$ & $4(66.6)$ & $1(50.0)$ & $49(52.6)$ & $69 / 132(52.2)$ & 0.304 \\
\hline \multicolumn{7}{|c|}{ TE findings for Non-NASH } \\
\hline TE LSM (kPa) & $5.88 \pm 2.01$ & $5.93 \pm 2.87$ & $4.66 \pm 2.44$ & $5.62 \pm 2.09$ & $5.84 \pm 2.06$ & 0.102 \\
\hline TE CAP $(\mathrm{dB} / \mathrm{m})$ & $202.91 \pm 80.5$ & $206.15 \pm 66.9$ & $208.053 \pm 55.2$ & $198.18 \pm 93.9$ & $201.04 \pm 90.3$ & 0.092 \\
\hline \multicolumn{7}{|l|}{ Liver histology for NASH } \\
\hline
\end{tabular}

Y: Year; M: Male; BMI: Body mass index; USG: Ultrasonography; TE: Transient elastography; LSM: Liver stiffness measure; CAP: Controlled attenuation parameter; NAS: NAFLD activity score. Results expressed as number (\%) or median (range) unless specified otherwise. P-value for comparison of all groups.

Table 5. The effects of coffee intake on liver enzymes and histology in patients with chronic alcohol consumption

\begin{tabular}{|c|c|c|c|c|c|}
\hline & Mild $(n=35)$ & Moderate $(n=11)$ & Heavy $(n=9)$ & Total $(n=55)$ & $\mathbf{p}$ \\
\hline Age $(Y)$ & $45.0 \pm 10.8$ & $46.6 \pm 12.4$ & $45.8 \pm 9.3$ & $45.2 \pm 10.3$ & 0.378 \\
\hline $\operatorname{Sex}[M(\%)]$ & $13(37.1)$ & $9(81.8)$ & $9(100.0)$ & $31(56.3)$ & 0.094 \\
\hline BMI $\left(\mathrm{kg} / \mathrm{m}^{2}\right)$ & $27.2 \pm 5.0$ & $27.1 \pm 4.9$ & $28.9 \pm 5.3$ & $27.3 \pm 5.3$ & 0.143 \\
\hline \multicolumn{6}{|l|}{ Laboratory } \\
\hline ALT (U/L) & $54.7 \pm 16.3$ & $60.2 \pm 13.7$ & $64.7 \pm 14.4$ & $56.1 \pm 15.1$ & 0.085 \\
\hline \multicolumn{6}{|l|}{ Coffee consumption } \\
\hline Non-Coffee drinker & $3(8.5)$ & $1(9.0)$ & $1(11.1)$ & $5(9.0)$ & 0.104 \\
\hline Occasional drinker & $16(45.7)$ & $3(27.2)$ & $2(22.2)$ & $21(38.1)$ & 0.266 \\
\hline Regular drinker $(<5 \mathrm{y})$ & $10(28.5)$ & $2(18.1)$ & $2(22.2)$ & $14(25.4)$ & 0.243 \\
\hline TE CAP $(\mathrm{dB} / \mathrm{m})$ & $206.30 \pm 79.8$ & $285.63 \pm 54.91$ & $313.55 \pm 46.83$ & $240.47 \pm 85.1$ & 0.003 \\
\hline
\end{tabular}

Y: Year; M: Male; BMI: Body mass index; USG: Ultrasonography; TE: Transient elastography; LSM: Liver stiffness measure; CAP: Controlled attenuation parameter. Results expressed as number (\%) or median (range) unless specified otherwise. P-value for comparison of all groups.

more coffee for a longer duration of time had lower liver enzyme levels. The mean serum ALT and AST levels were significantly lower in the regular coffee drinkers for more than five years group compared to other groups $(\mathrm{p}=0.028$ for ALT and $\mathrm{p}=0.042$ for AST). NAS and fibrosis scores were also lower in patients who drank more coffee for a longer period. Mean NAS and fibrosis scores of the patients with NASH were $5.01 \pm 0.41$ and $0.80 \pm 0.12$ for the regular coffee drinkers ( $>5$ years) group, $5.91 \pm 0.86$ and $2.03 \pm 0.42$ for non-coffee drinkers, $5.24 \pm 0.54$ and $1.92 \pm 0.19$ for occasional coffee drinkers, and $5.32 \pm 1.01$ and $1.04 \pm 0.36$ for regular coffee drinkers $<5$ years group. These differences were statistically significant for the fibro- sis score $(\mathrm{p}=0.045)$ but were not statistically significant for the NAS score (Table 3 ).

\section{Effects of Coffee Type on Liver Enzymes and Liver Histology}

The vast majority of the patients $n=93(70.4 \%)$ had a history of drinking a combination of Turkish, granulated and filtered coffee. There were 31 (23.5\%) only Turkish coffee drinkers, six only granulated coffee drinkers $(4.5 \%)$, and two only filtered coffee drinkers (1.6\%). Although the serum ALT and AST levels, LSM, CAP, NAS and fibrosis scores were lowest in the combination group, these differences and other study pa- 
Table 6. The effects of coffee intake on liver enzymes and histology in patients with chronic alcohol consumption

\begin{tabular}{|c|c|c|c|c|c|c|}
\hline & $\begin{array}{l}\text { Non-coffee } \\
\text { drinker } \\
(n=5)\end{array}$ & $\begin{array}{l}\text { Occasional } \\
\text { drinker } \\
(n=21)\end{array}$ & $\begin{array}{l}\text { Regular drinker } \\
(<5 \mathrm{yr}) \\
(\mathrm{n}=14)\end{array}$ & $\begin{array}{l}\text { Regular drinker } \\
(>5 \mathrm{yr}) \\
(\mathrm{n}=15)\end{array}$ & $\begin{array}{l}\text { Total } \\
(n=55)\end{array}$ & $\mathbf{p}$ \\
\hline Age (Y) & $42.3 \pm 9.1$ & $46.4 \pm 10.2$ & $44.0 \pm 8.3$ & $45.1 \pm 9.8$ & $45.2 \pm 10.3$ & 0.466 \\
\hline $\operatorname{Sex}[M(\%)]$ & $2(40.0)$ & $11(52.3)$ & $9(64.2)$ & $9(60.0)$ & $31(56.3)$ & 0.640 \\
\hline BMI $\left(\mathrm{kg} / \mathrm{m}^{2}\right)$ & $27.9 \pm 5.6$ & $28.0 \pm 5.8$ & $26.3 \pm 5.3$ & $25.4 \pm 6.6$ & $27.3 \pm 5.3$ & 0.180 \\
\hline \multicolumn{7}{|l|}{ Liver enzymes } \\
\hline ALT (U/L) & $70.1 \pm 24.2$ & $75.2 \pm 18.4$ & $35.8 \pm 11.7$ & $26.3 \pm 10.2$ & $56.1 \pm 15.1$ & 0.038 \\
\hline AST (U/L) & $60.7 \pm 14.6$ & $47.5 \pm 11.6$ & $41.0 \pm 11.1$ & $19.1 \pm 14.7$ & $45.8 \pm 11.6$ & 0.046 \\
\hline GGT (U/L) & $108.6 \pm 10.3$ & $112 \pm 16.9$ & $95.2 \pm 15.1$ & $89.0 \pm 10.1$ & $106.6 \pm 16.4$ & 0.035 \\
\hline Steatosis on USG & $4(80.0)$ & $9(42.8)$ & $5(35.7)$ & $3(20.0)$ & $21(38.1)$ & 0.020 \\
\hline \multicolumn{7}{|l|}{ TE Findings } \\
\hline TE LSM (kPa) & $6.98 \pm 3.90$ & $6.92 \pm 3.93$ & $6.08 \pm 3.31$ & $4.02 \pm 2.19$ & $6.49 \pm 3.86$ & 0.012 \\
\hline TE CAP $(\mathrm{dB} / \mathrm{m})$ & $283.504 \pm 71.6$ & $296.93 \pm 89.22$ & $232.05 \pm 58.03$ & $208.73 \pm 62.1$ & $240.47 \pm 85.1$ & 0.021 \\
\hline
\end{tabular}

Y: Year; M: Male; BMI: Body mass index; USG: Ultrasonography; TE: Transient elastography; LSM: Liver stiffness measure; CAP: Controlled attenuation parameter; NAS: NAFLD activity score. Results expressed as number (\%) or median (range) unless specified otherwise. P-value for comparison of all groups.

rameters such as age, gender, and BMI were not significant between the groups ( $\mathrm{p}>0.05)$ (Table 4).

\section{EffectsofCoffeein Patients with ChronicAlcoholConsumption}

There were 55 patients with a history of chronic alcohol consumption. Among these patients, 35 (63.6\%) were mild, 11 (20.0\%) were moderate and nine $(16.4 \%)$ were heavy drinkers. Age, gender, BMI, mean serum ALT, AST and GGT levels were similar between groups. Mean LSM scores for mild, moderate and heavy drinkers were $6.38 \pm 3.82$, $6.56 \pm 3.38$ and $6.88 \pm 3.14$ respectively $(\mathrm{p}=0.072)$. Mean CAP score was highest in the heavy drinkers and lowest in the mild drinkers' group [313.55 \pm 46.83 vs. $206.30 \pm 79.8(\mathrm{p}=0.023)]$ (Table 5).

The majority (91\%) of the patients were coffee drinkers. There were five (9\%) non-coffee drinkers, 21 (38.1) occasional drinkers, 14 (25.4\% regular drinkers $<5$ years and $15(27.2 \%)$ regular drinkers $>5$ years. The sex, age and BMI distributions were similar between the groups Mean serum ALT, AST and GGT levels were significantly lowest in the regular coffee drinkers for more than five years' group and highest in the non-coffee and occasional coffee drinker groups This difference in the mean serum ALT, AST and GGT levels between the groups were statistically significant ( $\mathrm{p}=0.038$ for ALT, $\mathrm{p}=0.046$ for AST and $\mathrm{p}=0.035$ for GGT).

The presence of steatosis with USG was $20 \%$ in the regular coffee drinkers $>5$ years group, $80 \%$ in the no-coffee drinking group, and $42.8 \%$ and $35.7 \%$ in the occasional drinker and regular drinker $<5$ years groups, respectively $(\mathrm{p}=0.020)$ (Table 6).

TE findings showed that mean LSM and CAP score was significantly lower in the regular coffee drinkers ( $>5$ years) group compared to other groups ( $\mathrm{p}=0.012$ for LSM score and $\mathrm{p}=0.021$ for CAP score). LSM scores for non-coffee drinkers, occasional drinkers' regular drinkers $<5$ years and regular drinkers $>5$ years were; $6.98 \pm 3.90,6.92 \pm 3.93$, $6.08 \pm 3.31$ and $4.02 \pm 2.19$ respectively $(\mathrm{p}=0.012)$. Meanwhile, the CAP scores in the same groups were $283.504 \pm 71.6,296.93 \pm 89.22$, $232.05 \pm 58.03$ and $208.73 \pm 62.1$ respectively $(\mathrm{p}=0.021)$ (Table 6$)$.

To investigate the effects of coffee on serum liver enzyme levels in patients with a history of chronic alcohol consumption, we first performed a univariate analysis on the factors that affected the serum ALT lev- els. Patients with normal liver enzymes were more likely to be female (75.0\% vs. $19.4 \%, p=0.033)$, consumed more amount of coffee for a longer period $(80.0 \%$ vs. $9.6 \%, p=0.008)$, had less amount of steatosis in the liver $(12.5 \%$ vs. $58.0 \%, \mathrm{p}=0.030)$ and consumed less amount of alcohol (4.2\% vs. $25.8 \%, \mathrm{p}=0.018)$, had lower LSM (6.01 \pm 3.91 vs. $6.87 \pm 3.37, \mathrm{p}=0.013)$ and CAP scores $(215.88 \pm 79$ vs. $1291.09 \pm 90.6$, $\mathrm{p}=0.015$ ). Meanwhile, age and BMI were similar between the two groups (Table 7a).

Cox regression analysis showed that daily amount of alcohol [OR: $3.12(1.18-5.25 ; 95 \% \mathrm{CI}),(\mathrm{p}=0.01)]$ and the amount, duration of coffee intake (daily for more than five years) [OR:0.74 (0.46-0.88;95\% $\mathrm{CI}),(\mathrm{p}=0.03)$ ] and LSM score [OR:1.68 $(0.94-3.07 ; 95 \% \mathrm{CI}),(\mathrm{p}=0.04)$ ] were the factors that had an impact on serum ALT levels, meanwhile gender and CAP score did not $(\mathrm{p}=0.84$ and $\mathrm{p}=0.69)$ (Table $7 \mathrm{~b})$.

\section{Discussion}

In this cross-sectional study of 259 patients, we found that both the amount and the duration of coffee consumption caused a decrease in liver enzyme levels and an improvement in liver histology in healthy individuals with no steatosis $(\mathrm{n}=85)$, healthy individuals with steatosis $(\mathrm{n}=73)$, patients with NASH $(\mathrm{n}=101)$ and also in patients with a history of chronic alcohol consumption $(\mathrm{n}=55)$.

The effects of coffee on liver enzyme levels have been reported in several studies. In a recent cross-sectional study from Japan, Ikeda et al. ${ }^{[24]}$ analyzed the effects of coffee on a large cohort of 12,020 Japanese patients. They reported that higher coffee consumption was associated with lower levels of liver enzymes, and the inverse associations between coffee and liver enzymes were more evident among those with higher alcohol consumption. The authors concluded that coffee consumption probably confers protection against an alcohol-related increase in liver enzymes. In another study from the USA, a dose-dependent inverse association was reported between coffee consumption and serum ALT levels in a cohort of 5944 adults. ${ }^{[17]}$ Our results are in concordance with the aforementioned studies. We found that not only in healthy individuals, but also in patients with liver steatosis, NASH and patients with a history of chronic alcohol consumption, coffee reduced 
Table 7a. Univariate analysis of factors that affect serum ALT level in patients with chronic alcohol consumption

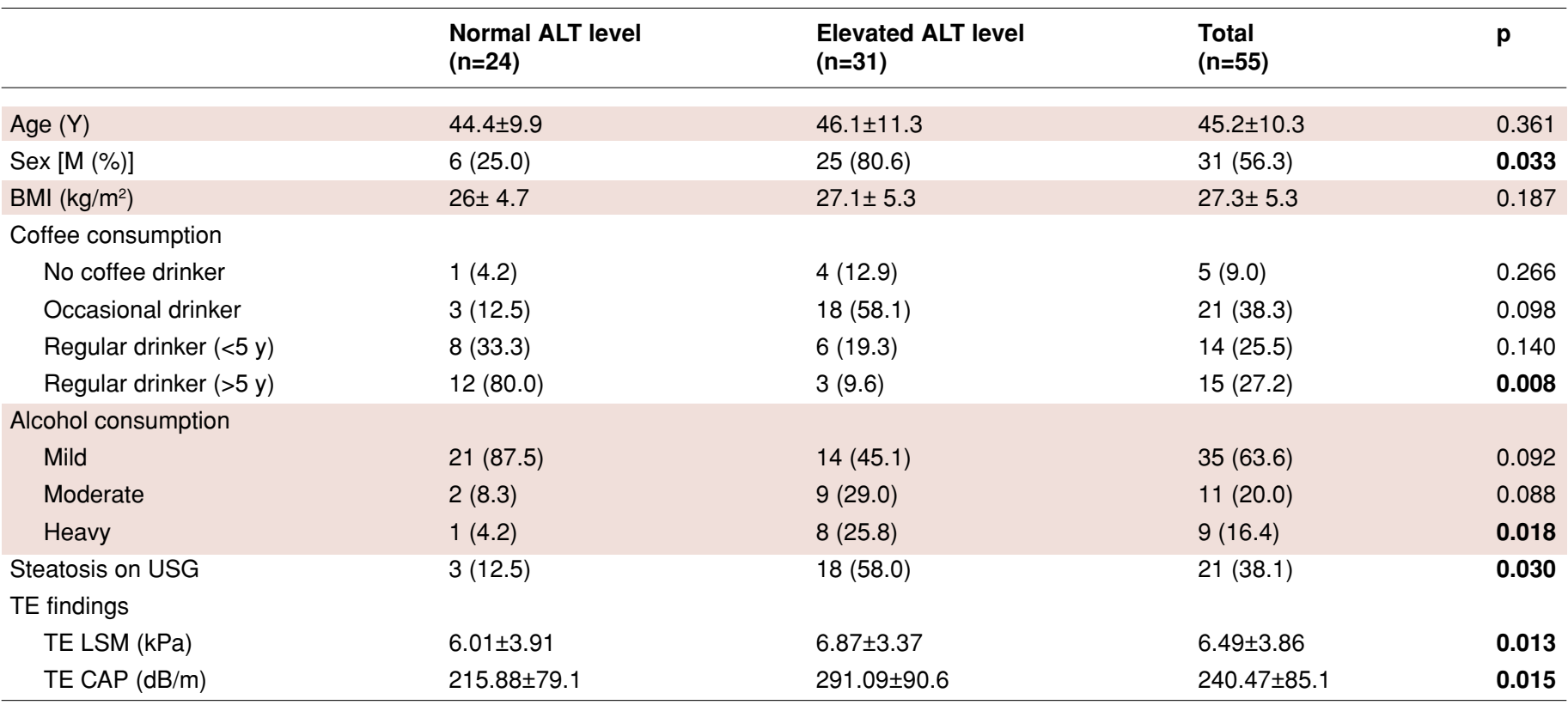

Y: Year; M: Male; BMI: Body mass index; USG: Ultrasonography; TE: Transient elastography; LSM: Liver stiffness measure; CAP: Controlled attenuation parameter. Results expressed as number (\%) or median (range) unless specified otherwise. P-value for comparison of all groups.

Table 7b. Multivariate analysis of factors that affect serum ALT level in patients with chronic alcohol consumption

\begin{tabular}{|c|c|c|c|}
\hline & $\begin{array}{l}\text { Univariate analysis } \\
\mathbf{p}\end{array}$ & $\begin{array}{l}\text { Multivariate analysis } \\
\text { Odds ratio }(95 \% \mathrm{Cl})\end{array}$ & $\mathbf{p}$ \\
\hline Heavy alcohol consumption (gr/day) & 0.018 & $3.12(1.18-5.25)$ & 0.01 \\
\hline TE LSM $(\mathrm{kPa})$ & 0.013 & $1.68(0.94-3.07)$ & 0.04 \\
\hline Gender (M) & 0.033 & $1.08(1.45-6.08)$ & 0.84 \\
\hline
\end{tabular}

Cl: Confidence interval; M: Male; TE: Transient elastography; LSM: Liver stiffness measure; CAP: Controlled attenuation parameter. Results expressed as number (\%) or median (range) unless specified otherwise.

the serum levels of ALT, AST and GGT. The reduction was more significant in patients who had regular daily coffee consumption for more than five years compared to the non-coffee drinkers group. Mean serum ALT and AST levels were 21.2 $\pm 11.7 \mathrm{U} / \mathrm{L}$ and 19.5 $\pm 10.4 \mathrm{U} / \mathrm{L}$ for regular coffee drinkers ( $>5$ years) group, $56.4 \pm 15.6 \mathrm{U} / \mathrm{L}$ and $42.6 \pm 15.2 \mathrm{U} / \mathrm{L}$ for non-coffee drinkers, $38.7 \pm 11.1 \mathrm{U} / \mathrm{L}$ and $32.9 \pm 14.9 \mathrm{U} / \mathrm{L}$ for occasional coffee drinkers, and $30.3 \pm 9.2 \mathrm{U} / \mathrm{L}$ and $25.2 \pm 11.6 \mathrm{U} / \mathrm{L}$ for regular coffee drinkers ( $<5$ year) group.

Previous studies on NASH and NAFLD patients have reported that coffee consumption reduces the amount of steatosis and fibrosis in a dose dependent manner. ${ }^{[10-13]}$ A recent study by Molloy et al. ${ }^{[17]}$ analyzed the effects of coffee amount on degree of liver steatosis and fibrosis. They found that when comparing patients with NASH stage $0-1$ to those with NASH stage $2-4$, there was a significant difference in coffee consumption $(p=0.016)$. Another interesting finding was that there was a negative relationship between the amount of coffee consumption and hepatic fibrosis $(r=-0.215 ; \mathrm{p}=0.035)$. In our study, we also found that the amount and duration of coffee consumption had a protective effect on liver histology. The NAS score and the fibrosis score were lowest in patients who drank coffee on a daily basis for more than five years (5.01 and 0.80) and highest in patients who never drank coffee (5.91 and 2.03). This hepatoprotective effect of coffee in liver disease, especially in NASH, NAFLD and alcoholic steatosis, is proposed to be mediated by the antioxidant, anti-inflammatory and metabolic effects of coffee, which are intervened not only by caffeine but also other ingredients of coffee such as cafestol, carveol, polyphenols and melanoids. ${ }^{[14-17]}$ Many studies have shown that coffee reduces reactive oxygen species and oxidized glutathione level, increases serum adiponectin level, decreases serum leptin, and saturated fatty acid level. Coffee also decreases cytokine levels, such as TNF-alpha, IFN-gamma, PPAR-alpha, IL-4 and IL-10, and has antifibrotic effect by decreasing TGF-beta and connective tissue growth factor levels. ${ }^{[14-17]}$

In this study, we found that the amount and duration of coffee consumption (daily for more than five years) [OR: $0.74(0.46-0.88 ; 95 \%$ $\mathrm{CI}),(\mathrm{p}=0.03)]$ was an independent factor for ALT normalization in alcoholic patients. Mean serum ALT and GGT levels were significantly lower in alcoholic patients who drank coffee on daily basis for 
more than five years $(\mathrm{p}=0.038$ for ALT and $\mathrm{p}=0.035$ for GGT). Studies investigating the role of coffee consumption on alcohol-related liver diseases have clearly reported that coffee reduces serum ALT and GGT levels in a dose dependent matter. ${ }^{[7,24-27]}$ The causal mechanistic explanation by which coffee exerts beneficial effect is still pending. Enhanced oxidative stress and inflammation are the key mechanism in the initiation of hepatotoxicity and GGT is an enzyme that is involved in the metabolism of glutahione which plays a crucial role in oxidative stress. In an interesting study by Wang et al., ${ }^{[25]}$ a rat alcoholic liver fibrosis model was designed. They found that caffeine significantly decreased ALT, AST, hyaluronic acid, laminin, N-terminal procollagen type III and type IV collagen levels and reversed liver fibrosis via cAMP/PKA/CREB signal pathway through adenosine A2A receptors in hepatic stellate cells.

Published data have shown that caffeine levels in a cup of coffee vary significantly due to variations in coffee blend, the exact amount of ground coffee used, and the brewing technique. In this study, we also investigated the effects of different types of coffee brewing techniques on liver diseases. Most of our patients $(70.4 \%)$ had a history of drinking a combination of Turkish coffee, granulated coffee and filtered coffee and there were a small number of patients in granulated $(n=6)$ and filtered coffee $(n=2)$ groups. Although the serum ALT and AST levels and NAS and fibrosis scores were lowest in the combination group, these differences were not significant between the groups ( $p>0.05)$. It is hard to come to a conclusion with these small numbers but based on these results, we believe that the duration and amount of daily coffee have more significant effects than coffee brewing type.

Our study has several strengths and limitations. The main limitations are (i) the amount of coffee consumption was based on patient reports, and for this reason, it is subject to recall bias. It was hard to calculate the exact amount of caffeine that was consumed by the participants and (ii) serum ALT, AST and GGT levels can fluctuate and can change over time. In our study, the study parameters were calculated at a single time point that may cause bias when interpreting the results. The strength of the study is its prospective design and the study population. To our knowledge, this is the only study that analyzes the effects of different types of coffee brewing techniques and the effects of long time coffee consumption on healthy individuals and those with different types of liver diseases, such as patients with NASH and patients with chronic alcohol consumption at the same study using liver biopsy and TE.

In summary, both the coffee amount and the duration of coffee consumption improve liver enzyme levels and liver histology in healthy individuals, in patients with chronic alcohol consumption, fatty liver and NASH. This result is more prominent in patients who drink coffee on a regular daily basis for more than five years.

Peer-review: Externally peer-reviewed.

Author Contributions: Concept - BD, NT, AGS, EG, YY, SY; Design - BD, NT, AGS, EG, UB, BC, YY, EV; Supervision - BD, NT, YY; Resource - BD, NT, AGS, SY, UB, EV, HUU, Materials - BD, SY, UB, EG, CBA, BC, YY; Data Collection and/or Processing - BD, AGS, EG, UB, BÇ, CBA, YY, EV, HUU; Analysis and/or Interpretation - BD, NT, YY, SY, UB, EG, EV, BC; Literature Search - BD, SY, UB, EG, AGS, HUU, EV; Writing - BD, NT, YY, SY; Critical Reviews - BD, NT, YY, SY, EV.

Conflict of Interest: The authors does mot have any conflict of interest.

Financial Disclosure: The study was supported by a grant from Marmara University Gastroenterology Institute Zeki Yollu Foundation.

\section{References}

1. Freedman ND, Park Y, Abnet CC, Hollenbeck AR, Sinha R. Association of coffee drinking with total and cause-specific mortality. N Engl J Med 2012;366(20):1891-904.

2. Ong A, Wong VW, Wong GL, Chan HL. The effect of caffeine and alcohol consumption on liver fibrosis - a study of 1045 Asian hepatitis B patients using transient elastography. Liver Int 2011;31(7):1047-1053.

3. La Vecchia C, Ferraroni M, Negri E, D’Avanzo B, Decarli A, Levi $\mathrm{F}$, et al. Coffee consumption and digestive tract cancers. Cancer Res 1989;49(4):1049-1051.

4. Kuper H, Tzonou A, Kaklamani E, Hsieh CC, Lagiou P, Adami HO, et al. Tobacco smoking, alcohol consumption and their interaction in the causation of hepatocellular carcinoma. Int J Cancer 2000;85(4):498-502.

5. Montella M, Polesel J, La Vecchia C, Dal Maso L, Crispo A, Crovatto M, et al. Coffee and tea consumption and risk of hepatocellular carcinoma in Italy. Int J Cancer 2007;120(7):1555-1559.

6. Ohishi W, Fujiwara S, Cologne JB, Suzuki G, Akahoshi M, Nishi N, et al. Risk factors for hepatocellular carcinoma in a Japanese population: a nested case-control study. Cancer Epidemiol Biomarkers Prev 2008;17(4):846854.

7. Hu G, Tuomilehto J, Pukkala E, Hakulinen T, Antikainen R, Vartiainen E, et al. Joint effects of coffee consumption and serum gamma-glutamyltransferase on the risk of liver cancer. Hepatology 2008;48(1):129-136.

8. Wakai K, Kurozawa Y, Shibata A, Fujita Y, Kotani K, Ogimoto I, et al; JACC Study Group. Liver cancer risk, coffee, and hepatitis $\mathrm{C}$ virus infection: a nested case-control study in Japan. Br J Cancer 2007;97(3):426-428.

9. Freedman ND, Curto TM, Lindsay KL, Wright EC, Sinha R, Everhart JE; HALT-C TRIAL GROUP. Coffee consumption is associated with response to peginterferon and ribavirin therapy in patients with chronic hepatitis $\mathrm{C}$. Gastroenterology 2011;140(7):1961-1969.

10. Gutiérrez-Grobe Y, Chávez-Tapia N, Sánchez-Valle V, Gavilanes-Espinar JG, Ponciano-Rodríguez G, Uribe M, et al. High coffee intake is associated with lower grade nonalcoholic fatty liver disease: the role of peripheral antioxidant activity. Ann Hepatol 2012;11(3):350-355.

11. Birerdinc A, Stepanova M, Pawloski L, Younossi ZM. Caffeine is protective in patients with non-alcoholic fatty liver disease. Aliment Pharmacol Ther 2012;35(1):76-82.

12. Molloy JW, Calcagno CJ, Williams CD, Jones FJ, Torres DM, Harrison SA. Association of coffee and caffeine consumption with fatty liver disease, nonalcoholic steatohepatitis, and degree of hepatic fibrosis. Hepatology 2012;55(2):429-436.

13. Catalano D, Martines GF, Tonzuso A, Pirri C, Trovato FM, Trovato GM. Protective role of coffee in non-alcoholic fatty liver disease (NAFLD). Dig Dis Sci 2010;55(11):3200-3206.

14. Wan CW, Wong CN, Pin WK, Wong MH, Kwok CY, Chan RY, et al. Chlorogenic acid exhibits cholesterol lowering and fatty liver attenuating properties by up-regulating the gene expression of PPAR- $\alpha$ in hypercholesterolemic rats induced with a high-cholesterol diet. Phytother Res 2013;27(4):545-551.

15. Murase T, Misawa K, Minegishi Y, Aoki M, Ominami H, Suzuki Y, et al. Coffee polyphenols suppress diet-induced body fat accumulation by downregulating SREBP-1c and related molecules in C57BL/6J mice. Am J Physiol Endocrinol Metab 2011;300(1):E122-133.

16. Cavin C, Holzhaeuser D, Scharf G, Constable A, Huber WW, Schilter B. Cafestol and kahweol, two coffee specific diterpenes with anticarcinogenic activity. Food Chem Toxicol 2002;40(8):1155-1163.

17. Ruhl CE, Everhart JE. Coffee and caffeine consumption reduce the risk of elevated serum alanine aminotransferase activity in the United States. Gastroenterology 2005;128(1):24-32.

18. Bedossa P. Pathology of non-alcoholic fatty liver disease. Liver Int 2017;37:85-89.

19. Degertekin B, Tozun N, Demir F, Soylemez G, Yapalı S, Bozkurt U, et al. 
Determination of the upper limits of normal serum alanine aminotransferase (ALT) level in healthy Turkish population. Hepatology Forum 2020;2(1):4447.

20. Yilmaz Y, Ergelen R, Akin H, Imeryuz N. Noninvasive detection of hepatic steatosis in patients without ultrasonographic evidence of fatty liver using the controlled attenuation parameter evaluated with transient elastography. Eur J Gastroenterol Hepatol 2013;25(11):1330-1334.

21. Tsochatzis EA, Gurusamy KS, Ntaoula S, Cholongitas E, Davidson BR, Burroughs AK. Elastography for the diagnosis of severity of fibrosis in chronic liver disease: a meta-analysis of diagnostic accuracy. J Hepatol 2011;54(4):650-659.

22. de Lédinghen V, Vergniol J, Foucher J, Merrouche W, le Bail B. Non-invasive diagnosis of liver steatosis using controlled attenuation parameter (CAP) and transient elastography. Liver Int 2012;32(6):911-918.

23. Kaya E, Bakır A, Eren F, Yılmaz Y. The utility of noninvasive scores in non-alcoholic fatty liver disease patients with normal and elevated serum transaminases. Hepatology Forum 2020;1(1):8-13.

24. Ikeda M, Maki T, Yin G, Kawate H, Adachi M, Ohnaka K, et al. Relation of coffee consumption and serum liver enzymes in Japanese men and women with reference to effect modification of alcohol use and body mass index. Scand J Clin Lab Invest 2010;70(3):171-179.

25. Wang Q, Dai X, Yang W, Wang H, Zhao H, Yang F, et al. Caffeine protects against alcohol-induced liver fibrosis by dampening the cAMP/PKA/CREB pathway in rat hepatic stellate cells. Int Immunopharmacol 2015;25(2):340352.

26. Setiawan VW, Wilkens LR, Lu SC, Hernandez BY, Le Marchand L, Henderson BE. Association of coffee intake with reduced incidence of liver cancer and death from chronic liver disease in the US multiethnic cohort. Gastroenterology 2015;148(1):118-125.

27. Danielsson J, Kangastupa P, Laatikainen T, Aalto M, Niemelä O. Dose- and gender-dependent interactions between coffee consumption and serum GGT activity in alcohol consumers. Alcohol Alcohol 2013;48(3):303-307. 